\section{Die Alkoholprävention: auch ein politisches Mandat für den Grundversorger}

Als Ärzte sind wir alle mit den durch Alkoholmissbrauch hervorgerufenen Problemen konfrontiert. Im Jahr 2000 starteten das Bundesamt für Gesundheit (BAG), die Schweizerische Fachstelle für Alkohol und andere Drogen (SFA) sowie die Eidgenössische Alkoholverwaltung (EAV), drei durch ihr langjähriges Engagement in der Alkoholprävention bedeutende Institutionen, gemeinsam die Nationale Kampagne «Alles im Griff?». Seither messen mehr und mehr Ärzte dem risikoreichen Alkoholkonsum die Bedeutung zu, die er verdient hat, nämlich jene eines prioritären Gesundheitsproblems.

Hinter der Kampagne steckt der politische Wille, die Prävention auf nationaler Ebene zu unterstützen, das Einführen neuer Strategien auf Gemeindeebene (Teilprojekt «Die Gemeinden handeln») und bei den Gesundheitsfachleuten (Teilprojekt «Fortbildung der Ärzte») zu erleichtern, sowie mit einer breit angelegten Medienkampagne durch Spots, Plakate und Internetspiel die Bevölkerung zu sensibilisieren. Drei Jahre sind indessen wenig, um die Faktoren des in der Schweiz so weit verbreiteten risikoreichen Alkoholkonsums entscheidend zu beeinflussen.

Die erste Phase des Alkoholpräventionsprogramms «Alles im Griff?» dauert noch bis Ende 2002 und wir wollten den diesbezüglichen Standpunkt von Frau Bundesrätin Ruth Dreifuss, Vorsteherin des EDI und demzufolge auch des Gesundheitswesens, kennenlernen.
So haben wir erfahren, dass der Bundesrat angesichts des vergleichsweise hohen Ausmasses der durch den Alkoholkonsum verursachten Probleme und der ersten positiven Ergebnisse von «Alles im Griff?» mehr Mittel zur Verfügung stellen will, um die Alkoholprävention im Bereich Risikokonsum fortzusetzen und $\mathrm{zu}$ intensivieren. Es tritt ebenfalls klar hervor, dass der Grundversorger als eine der Schlüsselfiguren in der Alkoholprävention betrachtet wird.

Von den von Frau Bundesrätin Dreifuss vorgestellten Elementen, die beim Arzt eine «Präventionskultur» fördern könnten, scheinen uns zwei besonders interessant zu sein: eine Fortbildung, welche die Qualität der Arzt-PatientBeziehung thematisiert, und die Anerkennung durch die massgeblichen Stellen, der Präventivleistung als spezifischer ärztlicher Tätigkeit - dies im Hinblick auf die Übernahme der über die Grundkonsultation hinausgehenden Leistungen durch die obligatorische Krankenversicherung.

Wir wussten es bereits: der Dialog mit unseren Patienten ist das Schlüsselelement für eine effiziente und respektvolle Prävention. Möglicherweise neu ist, dass wir von nun an ein politisches Mandat haben, um Präventionsprogramme rascher voranzutreiben und zu verbreiten.

Dr. med. Beat Stoll, MPH, FMH Innere Medizin Leiter des Teilprojektes «Fortbildung der Ärzte» IMSP, Universität Genf 


\section{La prévention de l'alcoolisme: un mandat politique pour les médecins de premier recours}

En tant que médecin, nous sommes tous confrontés aux problèmes qu'engendre la consommation abusive d'alcool. En 2000, l'Office fédéral de santé publique (OFSP), l'Institut Suisse de prévention d'alcool et d'autres toxicomanies (ISPA) et la Régie fédérale d'alcool (RFA), trois grandes institutions engagées de longue date dans la prévention alcool, se sont mis ensemble pour lancer la campagne nationale «Ça débouche sur quoi?». Dès lors, de plus en plus de médecins commencent à considérer la consommation d'alcool à risque comme un problème majeur de santé publique dans notre pays.

Derrière la campagne, il y a cette volonté politique de soutenir au niveau national la prévention en facilitant l'émergence de nouvelles stratégies au sein des communes («Les communes bougent»), auprès de professionnels de la santé (projet «Formation continue des médecins») et par une campagne grand public (spots, affiche et jeu Internet). Cependant, trois ans sont peu pour agir efficacement sur les déterminants entraînant une consommation d'alcool à risque aussi répandue que dans notre pays.

La première phase de «Ça débouche sur quoi?» arrivant à terme en fin 2002, nous avons voulu connaître la position en la matière de Mme la Conseillère fédérale Ruth Dreyfuss, cheffe du département de l'Intérieur et donc aussi de la santé:
Nous apprenons qu'en vu de l'importance en santé publique occupée par l'alcool comparé aux autres substances addictives et encouragé par les premiers résultats positifs de «Ça débouche sur quoi?», le Conseil fédéral veut consacrer d'avantage de moyens afin de poursuivre et d'intensifier la prévention d'alcool axée sur la consommation à risque. Le médecin de premier recours est considéré comme une personne clé dans la prévention d'alcool. Parmi les éléments présentés par Mme la Conseillère fédérale susceptibles de promouvoir une «culture de prévention» chez le médecin, deux nous semblent particulièrement intéressants: une formation continue qui met au centre la qualité de la relation médecinmalade et la reconnaissance par les services compétents de la prestation préventive en tant qu'acte médical spécifique afin de garantir une prise en charge par l'assurance obligatoire des soins en sus de la consultation de base.

Nous le savions déjà, le dialogue avec nos patients est l'élément clé pour une prévention efficace et respectueuse. Ce qui est peut-être nouveau, c'est que nous possédons désormais un mandat politique pour accélérer la mise en œuvre.

Dr Beat Stoll, MPH, FMH Médecine Interne Responsable du projet «Formation continue des médecins» IMSP, Université de Genève 


\section{TARMED-Info}

Mit dieser Ausgabe der Schweizerischen Ärztezeitung lancieren wir eine neue Artikelserie, die TARMED gewidmet ist. Ein Redaktionsteam, zusammengesetzt aus den führenden TARMEDExperten der FMH, hat sich bereit erklärt, in regelmässigen Abständen über den Stand der Dinge zu informieren und systematisch zu vordefinierten Standardthemen Bezug zu nehmen. $\mathrm{Zu}$ diesen Themen soll insbesondere auch dann Stellung genommen werden, wenn keine Neuigkeiten vorliegen. Eine solche sonst ungewöhnliche Art der Berichterstattung scheint für diesen übersensiblen Bereich unserer Standespolitik angebracht, um damit - endlich! - eine zuverlässige und transparente Informationspolitik zu garantieren. Konkret werden Sie in den Nummern 19, 23, 27, 31 ... unter der Rubrik «TARMED-Info» erfahren, ob sich in den Bereichen Tarifstruktur, KVG-Verhandlungen, UVGVerhandlungen und Schnittstellen etwas getan hat oder nicht. Sie können auch Fragen an die
TARMED-Redaktion richten, vorzugsweise via E-Mail tarmed@emh.ch; häufige und wichtige Fragen - FAQs - werden ebenfalls unter dieser Rubrik publiziert.

Es sei Historikern überlassen zu untersuchen, wie die Informationspolitik während der Entstehungsgeschichte von TARMED in strategische und taktische Überlegungen eingebunden wurde. Ich bin aber überzeugt, dass sich in Zukunft mit systematischen und zuverlässigen Informationen die Diskussionen um noch verbleibende Schwierigkeiten, die etwa mit der Einführung des Tarifs mit Garantie noch auf uns zukommen und die nicht zu verachten sind, wenigstens auf eine sachliche Ebene einschränken lassen. Es kann und darf dabei nicht übersehen werden, dass eine allgemeine Tarmüdigkeit vorherrscht und dass mit den noch verbleibenden Energien sehr sparsam umgegangen werden muss.

M. Trutmann

\section{TARMED-Info}

Dans ce numéro du Bulletin des médecins suisses, nous lançons une nouvelle série d'articles consacrée au TARMED. Une équipe rédactionnelle, composée des experts les plus éminents de la FMH en la matière, s'est mise à disposition pour informer régulièrement sur l'état des choses et prendre systématiquement position sur des thèmes définis au préalable. Une prise de position se fera également dans les cas où il n'y a pas de nouveautés à ces sujets. Une telle façon d'informer, qui est plutôt inhabituelle, nous semble néanmoins appropriée pour cette partie ultrasensible de notre politique professionnelle, afin de garantir une politique d'information plus transparente et fiable, attendue depuis longtemps. Concrètement, vous verrez dans les numéros 19, 23, 27, 31 ..., sous la rubrique «TARMED-Info», s'il y a du nouveau concernant la structure tarifaire, les négociations tarifaires, le tarif LAA et les «interfaces» ou s'il n'y a rien à signaler. Vous pouvez également adresser vos questions à la rédaction TARMED, de préférence par courrier électronique à tarmed@emh.ch; les questions les plus fréquentes et les plusimportantes - les FAQ seront également publiées sous cette rubrique.

Laissons aux historiens le soin d'examiner comment, pendant l'épopée du TARMED, la politique d'information a été intégrée aux réflexions stratégiques et tactiques. Mais je suis convaincu qu'à l'avenir il sera possible, grâce à des informations systématiques et fiables, de rester sur un niveau strictement objectif dans les discussions sur les difficultés qui nous attendent, p.ex. avec l'introduction du TARMED, et qu'il s'agit de ne pas ignorer. Nous ne pouvons et nous ne devons pas perdre de vue que tout le monde est las du TARMED et qu'il est impératif d'utiliser nos énergies restantes avec parcimonie.

M. Trutmann 